\title{
AMPERO-PHOTOMETRIC METHOD (APM) FOR THE DETERMINATION OF COLORED SOLUTION CONCENTRATION USING A LIGHT DEPENDENT RESISTOR (LDR)
}

\author{
Adham A. El-Zomrawy \\ Department of Chemistry, Faculty of Science, Al-Azhar University, Nasr City 11884, Cairo, Egypt
}

Corresponding Author: azomrawy@yahoo.com

\begin{abstract}
A simple, portable photometer depending on the intensity of the light that passes through a colored solution was designed. Through it, the measured light intensity parameter is converted to an electrical current density, which flow in a circuit of light dependent resistor (LDR). The suggested device is characterized by that it does not depend on the absorptivity at a specific wavelength, but depends on the intensity of the light passing through the sample only. The merits of this method are low power, high sensitivity, and very low cost. The proposed method is in excellent harmony with the determination of the concentration of colored solutions by spectrophotometr.
\end{abstract}

Keywards: Spectrophotometr, Concentration, Kinetic, LDR, LED.

\section{INTRODUCTION}

Colorimetric sensors are momentous because of their ability to analysis with the naked eyes [1]. Therefore, the development of colorimetric sensor materials is an interesting challenge.

Spectrophotometers are commonly used to determine concentrations of colored solutions [2-7], and follow-up decolorization when water was treated from pollutants such as Basic Orange, Methylene Blue, Methyl Orange, Eosin Yellowish [8], Ponceau 6R [9,10], cationic phenothiazine dye, anionic mono azo dye, cationic fluorescent dye [11], acid red 17 dye [12], Eosin Y [13], bromophenol dye [14], Reactive Blue-21, Reactive Red-141, Rhodamine-6G [15], methyl blue [16], and green dye [17]. Spectrophotometer works on the principle of measurement of absorption at a specified wavelength of the light by the solution. In spectrophotometer the source emits a light at all wavelengths of the UV and visible spectrum [18]. Via the mirrors system, the light is directed towards a monochromator that selects only a specific wavelength light. The vast majority of analytical measurements are currently performed under specialized laboratory conditions using bench-top instruments. This has led to the exploration of alternative strategies using simple, portable, and low-cost devices that can be developed for analytical measurements.

Light emitting diodes (LEDs) are durable, small size, low-cost, low-energy and highly efficient in terms of energy conversion (electric to light) [19]. LEDs are ideal for the development of optical devices, so LEDs are widely used in electronic devices, and many applications such as the illumination source for fiber optic sensors [20-23] and reflectometers [24,25].

Light dependent resistors (LDRs), are the light-sensitive devices that commonly used to articulate the presence or absence of light. In general, LDR is a sensor that decreases its resistance by increasing the amount of light falls on it. That means, the resistance value of LDR becomes larger in the dark, but when it is exposed to the light, the resistance decreases dramatically to the lowest value. On the other hand, in some electronic circuits, an integrated circuit (IC) is used to invers the general way of the role of LDR, in the sense that their resistance increases with the light falling increases [26].

LDRs are manufactured from semiconductors as light sensitive materials. The most common semiconductors used are cadmium sulfide, lead sulfide, germanium, silicon and gallium arsenide [27]. 
In this paper, a low-cost portable device has been constructed to determine the concentrations of the colored solutions. The suggested instrument uses LED as a light source, falling on the sample to measure its concentration. The sample absorbed part of the incident light, while the transmitted part is detected by LDR as a generated the electrical signal, wich can be measured by milliameter.

\section{MATERIALS AND METHODS}

\subsection{The device}

The device (which I have construted) was constructed in two parts positioned in the same plane. The first part consists of the light emitter diode (LED) as a light source, which connected to an external circuit supplies the required voltage, and with a variable potentiometer (10 $\mathrm{k} \Omega$ ) to control the intensity of the light. The second part contains the light dependent resistance (LDR), that is used for the measurement of the light intensity transmitted from the sample solution. LDR is located within an electronic circuit consisting of LM358 IC, ammeter, and resistors $(4.7 \mathrm{k} \Omega, 10$ $\mathrm{k} \Omega$, and $220 \Omega$ ), as shown in Fig. 1. A 9V DC battery converted to $5 \mathrm{~V}$ by voltage regulator (L7805), used as a power supply circuit.

The cuvette holder is simply a rectangular orifice that allows for a $1.0 \mathrm{~cm}$ cuvette to be positioned in it. The $1.0 \mathrm{~cm}$ cuvettes are the industry standard and can be obtained at low cost from many suppliers.

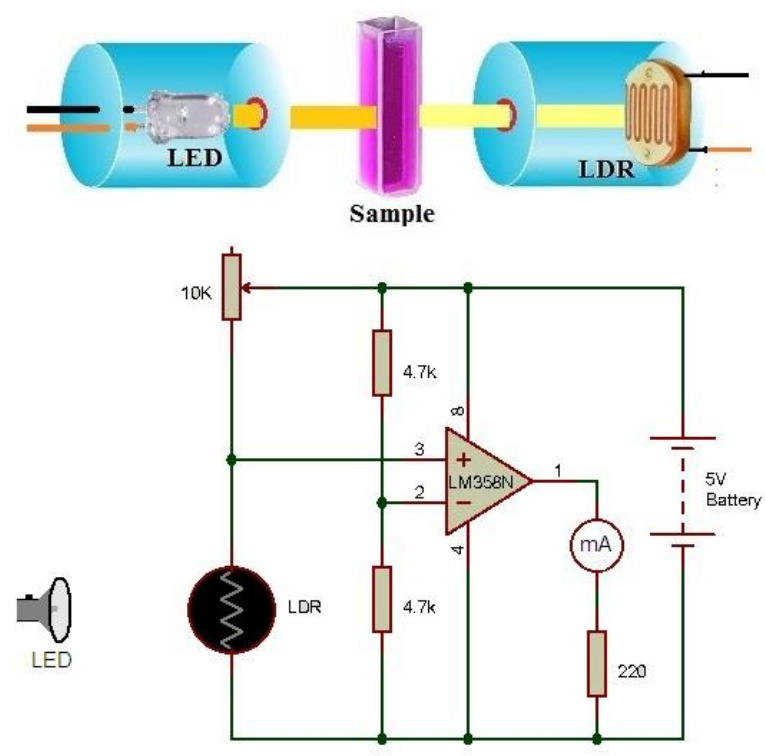

Fig. 1. Design and circuit of suggested device.

\subsection{Chemicals and reagents}

All the reagents used were of analytical grade without purification, potassium permanganate (PP), potassium dichromate (PDC), oxalic acid, and sulphuric acid were obtained from Al-Nasr pharmaceutical chemicals Co. Egypt, while Amaranth food dye (AFD) from Sigma-Aldrich.

The standard $1.0 \mathrm{mM}$ stock solutions of PP, PDC, and AFD were prepared by dissolving appropriate and exactly weighed a solid material in pre-calibrated $100 \mathrm{~cm}^{3}$ measuring flasks and diluted with double distilled water.

\subsection{Calibration method and sample determination}

The standard solutions of calibration curves were prepared in the concentration ranges 0.1 1.0, 0.1- 0.8, and 0.01-0.1 mMs of PP, PDC, and AFD respectively, by an appropriate dilution of the stock solutions.

For each standerd solution, was placed in the suggested device to adjust the resulted current passes in LDR circuit. After that, the light intensity of the LED adjusted to obtain a value of current slightly greater than zero using a variable resistor. The higher concentrations are placed sequentially, and current intensities are recorded for each solution. The same method is used to measure intensities of current for the concentrations of two other materials, and the unknown samples. 
For comparison, all of the above solutions were also measured by Perkin Elmer Lambda 35 spectrophotometer at 525,350 , and $520 \mathrm{~nm}$ of PP, PDC, AFD respectively.

\subsection{Kinetic studies}

A stock aqueous solutions $(100 \mathrm{ml})$ of both oxalic acid $(100 \mathrm{mM})$, and potassium permanganate $(10 \mathrm{mM})$ were prepared. Exactly $2.0 \mathrm{ml}$ of oxalic acid solution was transferred into a cuvette as a reactor, and $1.0 \mathrm{ml}$ of sulphuric acid $(1.0 \mathrm{~N})$ was added. Finally, 1.0 $\mathrm{ml}$ of potassium permanganate solution was added. $\mathrm{KMnO}_{4}$ reacts with $\mathrm{C}_{2} \mathrm{H}_{2} \mathrm{O}_{4}$ as follow:

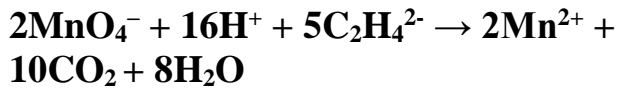

The reaction was followed at intervals by measuring the change in the colour of the permanganate solution by the proposed method, and the spectrophotometer.

\section{RESULTS AND DISCUSSION}

\subsection{Working of the Circuit}

The circuit is based on LM358 IC which is an operational amplifier (OP-AMP) with an operating voltage range of $3 \mathrm{~V}$ to $32 \mathrm{~V}$. The LDR is connected to the non-inverting terminal of the OP-AMP. The voltage across LDR changes with the intensity of transmitted light, and each time then the light was detected by the circuit it generates a high pule across the output, lead to decrease in the current intensity which passing in the circuit. The proposed device contains electronic components, so can be combined with a programmed microcontroller circuit to data processing and storage.

\subsection{Optical probe mechanism and light detection}

The amount of light that transfused through the colored solution and strikes the LDR is lead to changes in current intensity according to the concentration of each solution.

In the spectrophotometer should be chosen the wavelength compatible with the colored solution absorption spectrum to get optimal sensitivity $[7,28,29]$, whereas the proposed device is independent of the wavelength, but depends only on the light intensity passes through the colored solution, which falls upon LDR. The study involved a simple calibration of a change in color intensity according to the concentration of the solution.

\subsection{Calibration curves and samples determination}

The current intensity increases linearly with the concentration, depending on the LDR response to the intensity of the light which penetrates the colored solution. The graph is appeared direct proportional relationships between current intensity and absorbance versus the concentration of standard solutions (Fig. 2), These relationships represent the calibration curves, which achieves the BeerLambert law [3]:

$$
A=\varepsilon l c
$$




\begin{tabular}{c} 
Table 1. Analytical data and parameters of the proposed and spectrophotometric methods. \\
\cline { 3 - 8 }
\end{tabular}

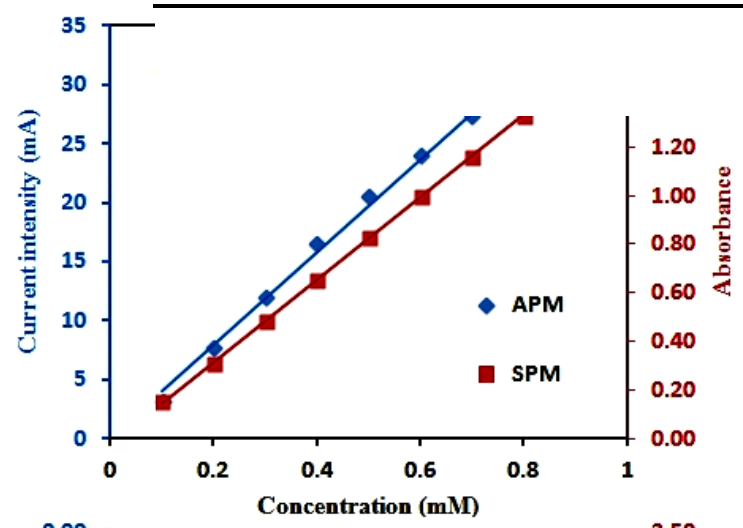

acquired from the designed system were in the harmony with those measured by spectrophotometer.

\subsection{Kinetic studies}

To evaluate how quickly the proposed apparatus responds to rapid changes in color solution concentrations, as well as the sensitivity and accuracy of the data resulting from the proposed method, the kinetics of the reaction between potassium permanganate and oxalic acid was studied. The presence of an excess of oxalic acid leads to the dependence of the reaction rate on the concentration of permanganate only, therefore, the pseudo first order equations was applied.

$\ln \left(I_{o}-I\right)=\ln I_{o}-k t \quad($ Proposed method $)$

$\ln \left(A_{o}-A\right)=\ln A_{o}-k t \quad$ (Spectrophotometer) $[9,10,12]$

Fig. 2. Calibration relationships between current intensity and absorbance $v s$ the concentration of standard solutions for AFD (a), PP (b), PDC (c)

According to the results obtained, simple modification can be made as follows:

$$
I=\varepsilon^{\prime} l c
$$

where $A$ the measured absorbance, $\varepsilon$ the molar absorptivity coefficient, $l$ the path length, and $c$ the analyte concentration, which derived from the spectrophotometer measurements, while $I$ the current intensity, and $\varepsilon^{\prime}$ the molar absorptivity coefficient, which derived from the proposed device measurements.

Samples of unknown concentration are analyzed by the suggested device and the results are accentuate in Table 1 . The results

where $A_{o}, A$ are initial absorbance and at time $t$ respectively, while $I_{0}, I$ the initial current intensity and at time intervals respectively.

Results were also compared with those measured from a spectrophotometer (Figs 3,4). The results showed that there is an excellent agreement between the two methods in the values of the rate constant of the reaction and the half-life period (Table 2).

According to the results obtained by studying the reaction speed, it was found that the halflife period is 17 seconds, which means that the concentration of the reaction decreases rapidly, and therefore the response of the LDR to the changes occurring is rapid. 
Table 2. Kinetic data of the rection between potasium permanganate against oxalic acid from the proposed and spectrophotometric methods.

\begin{tabular}{cccc}
\hline Method & $k_{1}\left(\mathrm{sec}^{-1}\right)$ & $t_{1 / 2}(\mathrm{sec})$ & $\mathbf{R}^{2}$ \\
\hline APM & 0.0409 & 17.0 & 0.9997 \\
SPM & 0.0410 & 16.9 & 0.9997 \\
\hline
\end{tabular}

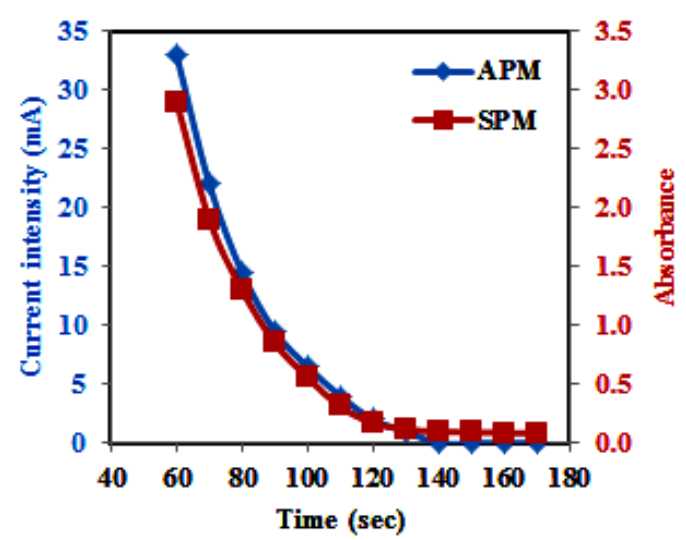

Fig. 3. Variation of current intensity of APM and absorption of SPM against time for the reaction between potasium permanganate with oxalic acid.

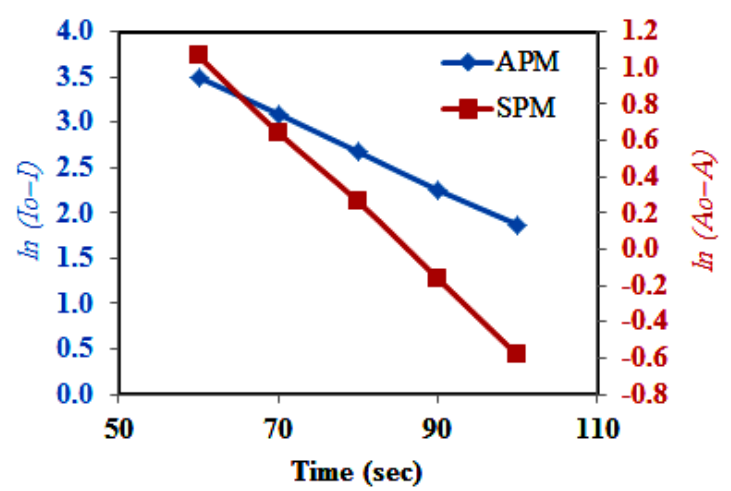

Fig. 4. Posedo first order kinetic reaction between potasium permanganate against oxalic acid from the proposed and spectrophotometric methods.

\section{CONCLUSION}

LDR is controlling the flow of current depending upon the light intensity, so it was used for measurement of the intensity of light passing through the colored solution, accordingly unknown concentration of the solution was determined. The LDR enters in an electronic circuit through which the current is increased when increasing the light falling on it from the colored solution. The results obtained from the suggested manner were compared with those acquired from the spectrophotometer and the results were in excellent agreement.

\section{REFERENCES}

[1] J. Chen, J. Pan, S. Chen, A naked-eye colorimetric sensor for $\mathrm{Hg} 2+$ monitoring with cascade signal amplification based on targetinduced conjunction of split DNAzyme fragments. Chemical Communications, 53(73) (2017) 10224-10227.

[2] E. K., Grasse, M. H. Torcasio, A. W. Smith. "Teaching UV-Vis spectroscopy with a 3Dprintable smartphone spectrophotometer." Journal of Chemical Education 93(1) (2016) 146-151.

[3] T. S. Kuntzleman, E. C. Jacobson. Teaching Beer's law and absorption spectrophotometry with a smart phone: a substantially simplified protocol. Journal of Chemical Education 93(7) (2016) 1249-1252.

[4] S. Taha, G. Rafat, F. Aboshosha, F. R. Mansour, A simple homemade spectrophotometer." Journal of analytical chemistry 72.2 (2017) 239-242.

[5] X. Yang, J. Ling, J. Peng, Q.E. Cao, Z.T. Ding, L.C. Bian, A colorimetric method for highly sensitive and accurate detection of iodide by finding the critical color in a color change process using silver triangular nanoplates, Anal. Chim. Acta 798 (2013) 74-81.

[6] C. Lei, X. Zhao, J. Sun, X. Yan, Y. Gao, H. Gao, S. Zhu, . H. Wang, A simple and novel colorimetric assay for tyrosinase and inhibitor screening using 3, 3', 5, 5'-tetramethylbenzidine as a chromogenic probe, Talanta 175 (2017) 457-462.

[7] A.A. El-Zomrawy, Selective and sensitive spectrophotometric method to determine trace amounts of copper metal ions using Amaranth food dye, Spectrochim. Acta Part A Mol. Biomol. Spectrosc. (2018) in press

[8] Y. Liu, J. Fu, S. Deng, X. Zhang, F. Shen, G. Yang, H. Peng, . Y. Zhang, Degradation of basic and acid dyes in high-voltage pulsed discharge, Journal of the Taiwan Institute of Chemical Engineers 45 (2014) 2480-2487.

[9] A. A. El-Zomrawy, Kinetic studies of photoelectrocatalytic degradation of Ponceau $6 \mathrm{R}$ dye with ammonium persulfate. Journal of Saudi Chemical Society 17 (2013) 397-402.

[10] S. M. Ahmed, A.A. El-Zomrawy, A.S.N. AlKamali, K.A.S. Ghaleb, Ponceau 6R dye decoloration and chromate reduction simultaneously in acid medium, Arabian Journal of Chemistry 8 (2015) 500-505. 
[11] M. Meena Kumari, D. Philip, Degradation of environment pollutant dyes using phytosynthesized metal nanocatalysts, Spectrochim. Acta Part A Mol. Biomol. Spectrosc. 135 (2015) 632-638.

[12] M. Thabet, A.A. El-Zomrawy, Degradation of acid red 17 dye with ammonium persulphate in acidic solution using photoelectrocatalytic methods, Arabian Journal of Chemistry 9 (2016) S204-S208.

[13] A. Hossain, A.B.M.S. Rayhan, M.J. Raihan, A. Nargis, I.M.I. Ismail, A. Habib, . A. Mahmood, Kinetics of Degradation of Eosin Y by One of the Advanced Oxidation Processes (AOPs) Fenton's Process, Am. J. Anal. Chem. 7 (2016) 863.

[14] S. Ameen, M.S Akhtar, H.S. Shin, Speedy photocatalytic degradation of bromophenol dye over ZnO nanoflowers, Mater. Lett. 209 (2017) 150-154.

[15] A. Vanamudan, M. Sadhu, P.S. Pamidimukkala, Nanostructured zirconium tungstate and its bionanocomposite with chitosan Wet peroxide photocatalytic degradation of dyes, Journal of the Taiwan Institute of Chemical Engineers 85 (2018) 74 82.

[16] J. Luan, Y. Zhuang, Synthesis, Structural Property, Photophysical Property, Photocatalytic Property of Novel $\mathrm{ZnBiErO}_{4}$ under Visible Light Irradiation, Materials 11 (2018) 303.

[17] R. Zhu, Y. Xu, Q. Bai, Z. Wang, X. Guo, . H. Kimura, Direct degradation of dyes by piezoelectric fibers through scavenging low frequency vibration, Chem. Phys. Lett. 702 (2018) 26-31.

[18] R. Morris, Spectrophotometry. Current Protocols Essential Laboratory Techniques, 11(1) (2015) 2.1.

[19] Kratkiewicz, Karl, et al. Review of Cost Reduction Methods in Photoacoustic Computed Tomography. arXiv preprint arXiv: (2019) 1902.09987.

[20] B. Mizaikoff, Infrared optical sensors for water quality monitoring. Water Sci. Technol. 47 (2003) 35-42.

[21] I. U. Primak, L.I. Sotskaya. Reconstruction of parameters of an adlayer on the surface of a channel optical waveguide. Opt. quantum electron. 35 (2003) 275-287.

[22] G. Hegedus, T. Sarkadi, T. Czigany, SelfSensing Polymer Composite: White-Light-
Illuminated Reinforcing Fibreglass Bundle for Deformation Monitoring. Sensors, 19(7) (2019) 1745.

[23] C. Sun, P. Lu, R. Wright, P. R. Ohodnicki, Low-cost fiber optic sensor array for simultaneous detection of hydrogen and temperature. In Fiber Optic Sensors and Applications Xv. International Society for Optics and Photonics, 10654 (2018) 1065405.

[24] F. A. A Matias, M. M. D. C. Vila, M. Tubino, A simple device for quantitative colorimetric diffuse reflectance measurements. Sensors and Actuators B Chemical 88 (2003) 60-66.

[25] M. H. Sorouraddin, M. Saadati, A simple fabrication of portable diffuse reflectometer for simultaneous analysis of common food dyes. Sensors and Actuators B: Chemical, 145(1) (2010) 367-372.

[26] M. M., PriyaDharsini, M. Keerthana, R. Keerthana, V. Malarvizhi, G. Meena, Smart Street Lighting. International Research Journal of Engineering and Technology, 3(4) (2016)1860-1868.

[27] R.P. Bauman, Absorption spectroscopy, John Wiley \& Sons, New York, (1962) 72-78.

[28] M. Basotra, S. K. Singh, M. Gulati, Development and validation of a simple and sensitive spectrometric method for estimation of cisplatin hydrochloride in tablet dosage forms: application to dissolution studies. ISRN Analytical Chemistry, 2013 (2013) 1-8.

[29] S. K. Pankaj, Z. Wan, W. Colonna, K. M. Keener, Effect of high voltage atmospheric cold plasma on white grape juice quality. Journal of the Science of Food and Agriculture, 97(12) (2017) 4016-4021.
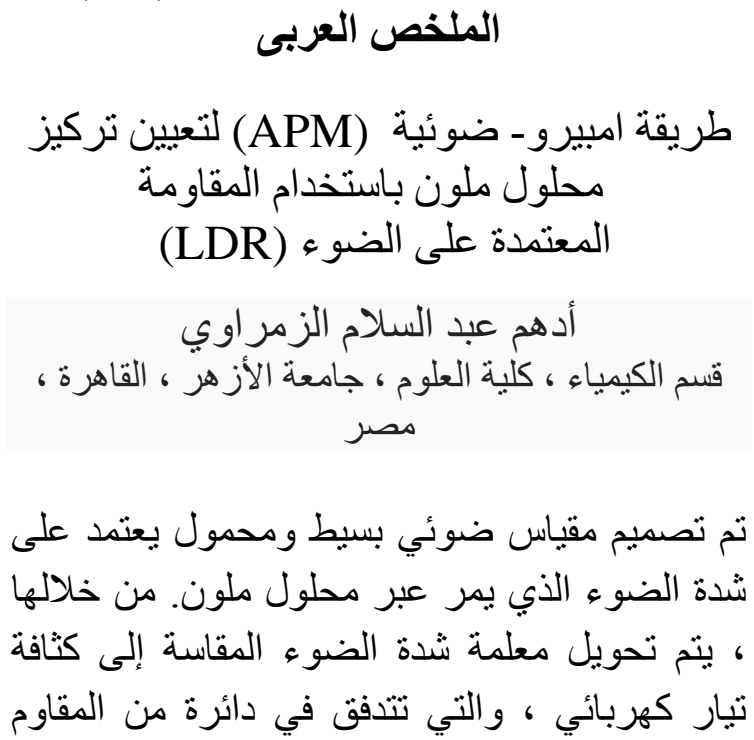
للضوء (LDR). يتميز الجهاز المقترح بأنه لا يعتمد على الامتصاصية عند طول موجة محدد ، ولكنه يعتمد على شدة الضوء الذي يمر عبه علئ العينة فقط.

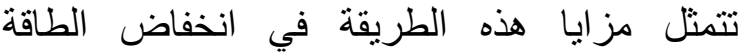

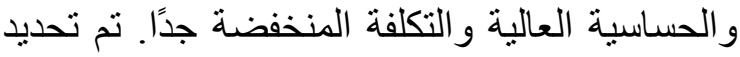
تركيز العديد من المحاليل الملونة بهذه الطريقة. ولتقييم استجابة الجهاز المقترح للتغير ات السريعة في

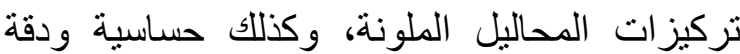
البيانات الناتجة عن الطريقة المقترحة ، تمت دراسة حركية التفاعل بين برمنجنات البوتاسيوم وحمض التهاته

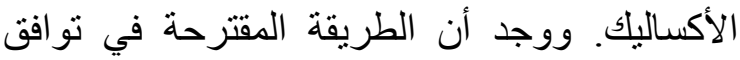

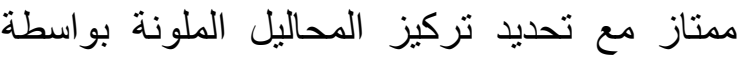
الطيف الضوئي. 